\title{
STARLIKENESS AND CONVEXITY OF A CLASS OF ANALYTIC FUNCTIONS
}

\author{
NIKOLA TUNESKI AND HÜSEYIN IRMAK
}

Received 4 July 2006; Revised 4 August 2006; Accepted 10 August 2006

Let $\mathscr{A}$ be the class of analytic functions in the unit disk that are normalized with $f(0)=$ $f^{\prime}(0)-1=0$ and let $-1 \leq B<A \leq 1$. In this paper we study the class $G_{\lambda, \alpha}=\{f \in \mathscr{A}$ : $\left.\left|\left(1-\alpha+\alpha z f^{\prime \prime}(z) / f^{\prime}(z)\right) / z f^{\prime}(z) / f(z)-(1-\alpha)\right|<\lambda, z \in \mathcal{U}\right\}, 0 \leq \alpha \leq 1$, and give sharp sufficient conditions that embed it into the classes $S^{*}[A, B]=\left\{f \in \mathscr{A}: z f^{\prime}(z) / f(z) \prec\right.$ $(1+A z) /(1+B z)\}$ and $K(\delta)=\left\{f \in \mathscr{A}: 1+z f^{\prime \prime}(z) / f^{\prime}(z) \prec(1-\delta)(1+z) /(1-z)+\delta\right\}$, where " $\prec$ " denotes the usual subordination. Also, sharp upper bound of $\left|a_{2}\right|$ and of the Fekete-Szegö functional $\left|a_{3}-\mu a_{2}^{2}\right|$ is given for the class $G_{\lambda, \alpha}$.

Copyright (c) 2006 Hindawi Publishing Corporation. All rights reserved.

\section{Introduction and preliminaries}

A region $\Omega$ from the complex plane $\mathbb{C}$ is called convex if for every two points $\omega_{1}, \omega_{2} \in \Omega$, the closed line segment $\left[\omega_{1}, \omega_{2}\right]=\left\{(1-t) \omega_{1}+t \omega_{2}: 0 \leq t \leq 1\right\}$ lies in $\Omega$. Fixing $\omega_{1}=0$ brings the definition of starlike region. If $\mathscr{A}$ denotes the class of functions $f(z)$ that are analytic in the unit disk $U=\{z:|z|<1\}$ and normalized by $f(0)=f^{\prime}(0)-1=0$, then a function $f \in \mathscr{A}$ is called convex or starlike if it maps $\mathcal{U}$ into a convex or starlike region, respectively. Corresponding classes are denoted by $K$ and $S^{*}$. It is well known that $K \subset S^{*}$, and it is well known that both are subclasses of the class of univalent functions and have the following analytical representations:

$$
\begin{gathered}
f \in K \Longleftrightarrow \operatorname{Re}\left(1+\frac{z f^{\prime \prime}(z)}{f^{\prime}(z)}\right)>0, \quad z \in \mathcal{U}, \\
f \in S^{*} \Longleftrightarrow \operatorname{Re} \frac{z f^{\prime}(z)}{f(z)}>0, \quad z \in \mathcal{U} .
\end{gathered}
$$

More about these classes may be found in [2].

Further, let $f, g \in \mathscr{A}$. Then we say that $f(z)$ is subordinate to $g(z)$, and we write $f(z) \prec$ $g(z)$, ifthere exists a function $\omega(z)$, analytic in the unit disk $U$, such that $\omega(0)=0$, 
$|\omega(z)|<1$, and $f(z)=g(\omega(z))$ for all $z \in \mathcal{U}$. Specially, if $g(z)$ is univalent in $\boldsymbol{U}$, then $f(z) \prec g(z)$ if and only if $f(0)=g(0)$ and $f(U) \subseteq g(u)$.

In terms of subordination, we have

$$
S^{*}=\left\{f \in \mathscr{A}: \frac{z f^{\prime}(z)}{f(z)} \prec \frac{1+z}{1-z}\right\}, \quad K=\left\{f \in \mathscr{A}: 1+\frac{z f^{\prime \prime}(z)}{f^{\prime}(z)} \prec \frac{1+z}{1-z}\right\} .
$$

If $-1 \leq B<A \leq 1$, then a generalization of class $S^{*}$ is

$$
S^{*}[A, B]=\left\{f \in \mathscr{A}: \frac{z f^{\prime}(z)}{f(z)} \prec \frac{1+A z}{1+B z}\right\} .
$$

Geometrically, this means that the image of $\mathcal{u}$ by $z f^{\prime}(z) / f(z)$ is inside the open disk centered on the real axis with diameter endpoints $(1-A) /(1-B)$ and $(1+A) /(1+B)$. Special selection of $A$ and $B$ leads us to the following classes: $S^{*}[1,-1] \equiv S^{*}, S^{*}[1-$ $2 \alpha,-1] \equiv S^{*}(\alpha)$-class of starlike functions of order $\alpha, 0 \leq \alpha<1$, and $K(\alpha)$ is the class of convex functions of order $\alpha, 0 \leq \alpha<1$, defined by $f(z) \in K(\alpha)$ if and only if $z f^{\prime}(z) \in$ $S^{*}(\alpha)$, that is,

$$
\operatorname{Re}\left(1+\frac{z f^{\prime \prime}(z)}{f^{\prime}(z)}\right)>\alpha, \quad z \in \mathcal{U} .
$$

These classes are widely studied during the past decades, mainly in two different directions: for developing criteria for starlikeness or convexity and for obtaining properties of the Maclaurin coefficients of a starlike or convex function. In this paper sufficient conditions (some of them sharp) that embed the class

$$
G_{\lambda, \alpha}=\left\{f \in \mathscr{A}:\left|\frac{1-\alpha+\alpha z f^{\prime \prime}(z) / f^{\prime}(z)}{z f^{\prime}(z) / f(z)}-(1-\alpha)\right|<\lambda, z \in \mathcal{U}\right\},
$$

$0<\alpha \leq 1, \lambda>0$, into the classes $S^{*}[A, B]$ and $K(\delta), 0 \leq \delta<1$, will be given, together with sharp upper bound of the Fekete-Szegö functional $\left|a_{3}-\mu a_{2}^{2}\right|, \mu \in \mathbb{R}$. Sufficient motivation for studying the class $G_{\lambda, \alpha}$ is the fact that it makes close connection between classes,

$$
\begin{gathered}
G_{\lambda, 1 / 2}=\left\{f \in \mathscr{A}:\left|\frac{1+z f^{\prime \prime}(z) / f^{\prime}(z)}{z f^{\prime}(z) / f(z)}-1\right|<2 \lambda, z \in \mathcal{U}\right\}, \\
G_{\lambda, 1}=\left\{f \in \mathscr{A}:\left|\frac{f(z) f^{\prime \prime}(z)}{f^{\prime 2}(z)}\right|<\lambda, z \in \mathcal{U}\right\}, \\
G_{\lambda, 1 /(2-\gamma)}=\left\{f \in \mathscr{A}:\left|\frac{1-\gamma+z f^{\prime \prime}(z) / f^{\prime}(z)}{z f^{\prime}(z) / f(z)}-(1-\gamma)\right|<\lambda(2-\gamma), z \in \mathcal{U}\right\},
\end{gathered}
$$

studied in $[1,6-9,11-13]$ and other references.

\section{Conditions for starlikeness and convexity}

For obtaining the result for convexity and starlikeness of the class $G_{\lambda, \alpha}$, we will use the method of differential subordinations. Valuable reference on this topic is [5]. The general theory of differential subordinations, as well as the theory of first-order differential 
subordinations, was introduced by Miller and Mocanu in $[3,4]$. Namely, if $\phi: \mathbb{C}^{2} \rightarrow \mathbb{C}$ is analytic in a domain $D$, if $h(z)$ is univalent in $\mathcal{U}$, and if $p(z)$ is analytic in $\mathcal{U}$ with $\left(p(z), z p^{\prime}(z)\right) \in D$ when $z \in \mathcal{U}$, then $p(z)$ is said to satisfy a first-order differential subordination if

$$
\phi\left(p(z), z p^{\prime}(z)\right) \prec h(z) .
$$

The univalent function $q(z)$ is said to be a dominant of the differential subordination (2.1) if $p(z) \prec q(z)$ for all $p(z)$ satisfying (2.1). If $\tilde{q}(z)$ is a dominant of $(2.1)$ and $\tilde{q}(z) \prec q(z)$ for all dominants of (2.1), then we say that $\widetilde{q}(z)$ is the best dominant of the differential subordination (2.1).

From the theory of first-order differential subordinations, we will make use of the following lemma.

Lemma 2.1 (see [4]). Let $q(z)$ be univalent in the unit disk $q$, and let $\theta(\omega)$ and $\phi(\omega)$ be analytic in a domain $D$ containing $q(u)$, with $\phi(\omega) \neq 0$ when $\omega \in q(\mathcal{U})$. Set $Q(z)=$ $z q^{\prime}(z) \phi(q(z)), h(z)=\theta(q(z))+Q(z)$, and suppose that

(i) $Q(z) \in S^{*}$;

(ii) $\operatorname{Re}\left(z h^{\prime}(z) / Q(z)\right)=\operatorname{Re}\left\{\theta^{\prime}(q(z)) / \phi(q(z))+z Q^{\prime}(z) / Q(z)\right\}>0, z \in \mathcal{U}$.

If $p(z)$ is analytic in $U$, with $p(0)=q(0), p(U) \subseteq D$, and

$$
\theta(p(z))+z p^{\prime}(z) \phi(p(z)) \prec \theta(q(z))+z q^{\prime}(z) \phi(q(z))=h(z),
$$

then $p(z) \prec q(z)$, and $q(z)$ is the best dominant of (2.2).

In the beginning, using Lemma 2.1 we will prove the following result.

Theorem 2.2. Let $f \in \mathscr{A},-1 \leq B<A \leq 1$, and $(1+|A|) /(3+|A|) \leq \alpha \leq 1$. If

$$
\frac{1-\alpha+\alpha z f^{\prime \prime}(z) / f^{\prime}(z)}{z f^{\prime}(z) / f(z)} \prec \alpha+(1-2 \alpha) \frac{1+B z}{1+A z}+\frac{\alpha z(A-B)}{(1+A z)^{2}} \equiv h(z),
$$

then $f \in S^{*}[A, B]$. This result is sharp.

Proof. We choose $p(z)=f(z) / z f^{\prime}(z), q(z)=(1+B z) /(1+A z), \theta(\omega)=(1-2 \alpha) \omega+\alpha$, and $\phi(\omega)=-\alpha$. Then $q(z)$ is convex, thus univalent, because $1+z q^{\prime \prime}(z) / q^{\prime}(z)=(1-$ $A z) /(1+A z) ; \theta(\omega)$ and $\phi(\omega)$ are analytic in the domain $D=\mathbb{C}$ which contains $q(U)$ and $\phi(\omega)$ when $\omega \in q(\mathcal{U})$. Further,

$$
Q(z)=z q^{\prime}(z) \phi(q(z))=\frac{\alpha(A-B) z}{(1+A z)^{2}}
$$

is starlike because $z Q^{\prime}(z) / Q(z)=(1-A z) /(1+A z)$. Further,

$$
\begin{gathered}
h(z)=\theta(q(z))+Q(z)=\alpha+(1-2 \alpha) \frac{1+B z}{1+A z}+\frac{\alpha z(A-B)}{(1+A z)^{2}}, \\
\operatorname{Re} \frac{z h^{\prime}(z)}{Q(z)}=\operatorname{Re}\left(1-\frac{1}{\alpha}+\frac{2}{1+A z}\right)>1-\frac{1}{\alpha}+\frac{2}{1+|A|},
\end{gathered}
$$


4 Starlikeness and convexity of analytic functions

$z \in \mathcal{U}$, which is greater or equal to zero if and only if $\alpha \geq(1+|A|) /(3+|A|)$. Therefore from Lemma 2.1, it follows that $p(z) \prec q(z)$, that is, $f \in S^{*}[A, B]$.

The result is sharp as the functions $z e^{A z}$ and $z(1+B z)^{A / B}$ show in the cases $B=0$ and $B \neq 0$, respectively.

Remark 2.3. According to the definition of subordination, the sharpness of the result of Theorem 2.2 means that $h(\mathcal{U})$ is the greatest region in the complex plane with the property that if

$$
\frac{1-\alpha+\alpha z f^{\prime \prime}(z) / f^{\prime}(z)}{z f^{\prime}(z) / f(z)} \in h(U)
$$

for all $z \in \mathcal{U}$, then $f(z) \in S^{*}[A, B]$.

The following corollary gives sharp sufficient conditions that embed $G_{\lambda, \alpha}$ into $S^{*}[A, B]$.

Corollary 2.4. Let $-1 \leq B<A \leq 1$ and $(1+|A|) /(3+|A|) \leq \alpha \leq 1$. Then

$$
\lambda=(A-B) \cdot \frac{(1-2 \alpha)|A|-(1-3 \alpha)}{(1+|A|)^{2}}
$$

is the greatest number such that $G_{\lambda, \alpha} \subseteq S^{*}[A, B]$.

Proof. In order to prove this corollary, due to Theorem 2.2 it is enough to show that

$$
\lambda=\min \{|h(z)-(1-\alpha)|:|z|=1\} \equiv \hat{\lambda}
$$

where $h(z)$ is defined as in the statement of the theorem and

$$
h(z)-(1-\alpha)=-z(A-B) \cdot \frac{A(1-2 \alpha) z+1-3 \alpha}{(1+A z)^{2}} .
$$

Further, let

$$
\begin{aligned}
\psi(t) & \equiv\left|h\left(e^{i \gamma \pi / 2}\right)-(1-\alpha)\right|^{2} \\
& =(A-B)^{2} \cdot \frac{\left[(1-2 \alpha)^{2} A^{2}+2(1-3 \alpha)(1-2 \alpha) A t+(1-3 \alpha)^{2}\right]}{\left(1+2 A t+A^{2}\right)^{2}},
\end{aligned}
$$

$t=\cos (\gamma \pi / 2) \in[-1,1]$. Thus $\hat{\lambda}=\min \{\sqrt{\psi(t)}:-1 \leq t \leq 1\}$.

If $\alpha \leq 1 / 2$, then $1-2 \alpha \geq 0$ and having in mind that $1-3 \alpha \leq-2|A| /(3+|A|) \leq 0$, we receive that $\psi(t)$ is a monotone function and

$$
\hat{\lambda}=\min \{\sqrt{\psi(-1)}, \sqrt{\psi(1)}\}=\min \{|h(-1)-(1-\alpha)|,|h(1)-(1-\alpha)|\}=\lambda .
$$

The last equality holds because $1-3 \alpha \pm A(1-2 \alpha) \geq 0$ is equivalent to $\alpha \geq(1+|A|) /$ $(3+|A|) \geq(1-|A|) /(3-2|A|)$.

If $\alpha>1 / 2$, we have the following analysis. Equation $\psi_{t}^{\prime}(t)=0$ has unique solution

$$
t_{*}=-\frac{A^{2}(1-\alpha)(1-2 \alpha)+(1-3 \alpha)(1-4 \alpha)}{2 A(1-2 \alpha)(1-3 \alpha)} .
$$


It can be verified that $\left|t_{*}\right|>1$ is equivalent to

$$
\varphi(A, \alpha) \equiv A^{2}(1-\alpha)(1-2 \alpha)-2|A|(1-2 \alpha)(1-3 \alpha)+(1-3 \alpha)(1-4 \alpha)>0 .
$$

Now, $\varphi(A, \alpha)$ is a decreasing function of $|A| \in[0,1]$ which implies that $\varphi(A, \alpha) \geq \varphi(1, \alpha)=$ $2 \alpha^{2}>0$. Thus, $\left|t_{*}\right|>1$, which implies that $\psi(t)$ is a monotone function on $[-1,1]$ leading to $\hat{\lambda}=\min \{\sqrt{\psi(t)}:-1 \leq t \leq 1\}=\min \{\sqrt{\psi(-1)}, \sqrt{\psi(1)}\}=\min \{|h(-1)-(1-\alpha)|$, $|h(1)-(1-\alpha)|\}$. At the end, the function

$$
\eta(A, \alpha) \equiv|h(1)-(1-\alpha)|-|h(-1)-(1-\alpha)|=2 A \cdot \frac{1-A^{2}-2 \alpha\left(2-A^{2}\right)}{(1+A)^{2}(1-A)^{2}}
$$

has the opposite sign of the sign of coefficient $A$. Therefore,

$$
\hat{\lambda}=\left\{\begin{array}{lc}
|h(1)-(1-\alpha)|, & A \geq 0 \\
|h(-1)-(1-\alpha)|, & A<0
\end{array}\right\}=\lambda
$$

Sharpness of the result follows from the sharpness of Theorem 2.2 (see Remark 2.3) and the fact that the obtained $\lambda$ is the greatest, which embeds the disk $|\omega-(1-\alpha)|<\lambda$ in $h(u)$.

The following example exhibits some concrete conclusions that can be obtained from the results of the previous section by specifying the values $\alpha, A, B$.

Example 2.5. Let $-1 \leq B<A \leq 1$.

(i) $G_{\lambda, 1 / 2} \subseteq S^{*}[A, B]$ when $\lambda=(A-B) / 2(1+|A|)^{2}$.

(ii) $G_{\lambda, 1} \subseteq S^{*}[A, B]$ when $\lambda=(A-B) \cdot(2-|A|) / 2(1+|A|)^{2}$.

(iii) $G_{\lambda, 1 /(2-\gamma)} \subseteq S^{*}[A, B]$ when $\gamma \geq-(1-|A|) /(1+|A|)$ and $\lambda=(A-B) \cdot(1+\gamma-$ $\gamma|A|) / 2(1+|A|)^{2}$.

(iv) $G_{\lambda, \alpha} \subseteq S^{*}$ when $1 / 2 \leq \alpha \leq 1$ and $\lambda=\alpha / 2$.

(v) $G_{\lambda, \alpha} \subseteq S^{*}[0, B] \subset S^{*}(1 /(1-B))$ when $1 / 3 \leq \alpha \leq 1,-1 \leq B<0$ and $\lambda=B(1-3 \alpha)$. The value of $\lambda$ in each of the above cases is the greatest that makes the corresponding inclusion true.

Remark 2.6. The result from Example 2.5(i) is the same as in [13, Corollary 2.6]. Also, for $\alpha=1 / 2$ in Example 2.5(v), we receive the same result as in [6, Theorem 1]. Finally, for $\alpha=1$ and $B=-1$ in Example 2.5(v), we receive the same result as in [11, Corollary 2].

Next theorem studies connection between $G_{\lambda, \alpha}$ and the class of convex functions of some order.

Theorem 2.7. $G_{\lambda, \alpha} \subseteq K(2-1 / \alpha)$ when $1 / 2 \leq \alpha<1$ and $\lambda=(1-\alpha)(3 \alpha-1) / \sqrt{2\left(5 \alpha^{2}-4 \alpha+1\right)}$. Proof. Let $f \in G_{\lambda, \alpha}$ and $B=\lambda /(1-3 \alpha)$. Then, by Example 2.5(v) we have $f \in S^{*}[0, B]$, that is, $\left|f(z) / z f^{\prime}(z)-1\right|<B, z \in \mathcal{U}$. Further,

$$
1+\frac{z f^{\prime \prime}(z)}{f^{\prime}(z)}-\left(2-\frac{1}{\alpha}\right)=\frac{z f^{\prime}(z)}{\alpha f(z)} \cdot \frac{1-\alpha+\alpha z f^{\prime \prime}(z) / f^{\prime}(z)}{z f^{\prime}(z) / f(z)},
$$


6 Starlikeness and convexity of analytic functions

and for all $z \in \mathcal{U}$, we obtain

$$
\begin{aligned}
\left|\arg \left(1+\frac{z f^{\prime \prime}(z)}{f^{\prime}(z)}-2+\frac{1}{\alpha}\right)\right| & \leq\left|\arg \frac{z f^{\prime}(z)}{f(z)}\right|+\left|\arg \frac{1-\alpha+\alpha z f^{\prime \prime}(z) / f^{\prime}(z)}{z f^{\prime}(z) / f(z)}\right| \\
& \leq \arcsin |B|+\arcsin \frac{\lambda}{1-\alpha} \\
& =\arcsin \left(\frac{\lambda}{1-\alpha} \sqrt{1-B^{2}}+|B| \sqrt{1-\frac{\lambda^{2}}{(1-\alpha)^{2}}}\right) \\
& =\arcsin 1=\frac{\pi}{2},
\end{aligned}
$$

that is, $f \in K(2-1 / \alpha)$.

Example 2.8. For $\alpha=1 / 2$ and $\alpha=1 /(2-\gamma)$ in the previous theorem, we get

(i) $G_{\lambda, 1 / 2} \subseteq K$ when $\lambda=\sqrt{2} / 4$;

(ii) $G_{\lambda, 1 /(2-\gamma)} \subseteq K(\gamma)$ when $0 \leq \gamma<1$ and $\lambda=\left(1-\gamma^{2}\right) /\left[(2-\gamma) \sqrt{2\left(1+\gamma^{2}\right)}\right]$.

Remark 2.9. By putting $\alpha=1 /(2-\gamma), 0 \leq \gamma<1$, we get the result from [10, Theorem 2].

\section{Sharp estimate of the Fekete-Szegö functional}

In this section we give sharp estimates of $\left|a_{2}\right|$ and of the Fekete-Szegö functional $\mid a_{3}-$ $\mu a_{2}^{2} \mid$ for a function $f \in G_{\lambda, \alpha}$. We will use following lemmas.

LeMma 3.1 [2, page 41]. Let $p \in \mathscr{P}$, that is, let $p$ be analytic in $\mathcal{U}$, be given by $p(z)=$ $1+\sum_{n=1}^{\infty} p_{n} z^{n}$ and $\operatorname{Re} p(z)>0$ for $z \in \mathcal{U}$. Then $\left|p_{n}\right| \leq 2$ and for all $n \in \mathbb{N},\left|p_{2}-p_{1}^{2} / 2\right| \leq$ $2-\left|p_{1}\right|^{2} / 2$.

LEMMA 3.2. Let $\omega(z)=\sum_{n=1}^{\infty} c_{n} z^{n}$ be an analytic function in the unit disk $u$ and $|\omega(z)|<1$, $z \in \mathcal{U}$. Then $\left|c_{1}\right| \leq 1$ and $\left|c_{2}\right| \leq 1-\left|c_{1}\right|^{2}$.

Proof. Define a function $p(z)=1+\sum_{n=1}^{\infty} p_{n} z^{n} \in \mathscr{P}$ by $p(z)=(1-\omega(z)) /(1+\omega(z))$. Then $c_{1}=-p_{1} / 2, c_{2}=\left(p_{1}^{2} / 2-p_{2}\right) / 2$ and the rest follows from Lemma 3.1.

Theorem 3.3. Let $f(z)=z+\sum_{n=2}^{\infty} a_{n} z^{n} \in G_{\lambda, \alpha}$ for some $\lambda>0$ and $0 \leq \alpha \leq 1$. Then $\left|a_{2}\right| \leq$ $\lambda /|1-3 \alpha|$ and for any complex $\mu$, the following bound is sharp:

$$
\left|a_{3}-\mu a_{2}^{2}\right| \leq \max \left\{\frac{\lambda}{2|4 \alpha-1|}, \frac{\lambda^{2}|1-\mu|}{(1-3 \alpha)^{2}}\right\}
$$

Proof. If $f \in G_{\lambda, \alpha}$, then

$$
(1-\alpha) f(z) f^{\prime \prime}(z)+\alpha z f(z) f^{\prime \prime}(z)=z f^{\prime 2}(z)[1-\alpha+\lambda \omega(z)]
$$

where $\omega(z)=\sum_{n=1}^{\infty} c_{n} z^{n}$ is such that $|\omega(z)|<1, z \in \mathcal{U}$. After equating the coefficients, we get $a_{2}=\lambda c_{1} /(3 \alpha-1)$ and

$$
a_{3}=\frac{\lambda c_{2}}{2(4 \alpha-1)}+\frac{\lambda^{2} c_{1}^{2}}{(1-3 \alpha)^{2}}
$$


From Lemma 3.2, we get $\left|a_{2}\right| \leq \lambda /|1-3 \alpha|$. Further,

$$
a_{3}-\mu a_{2}^{2}=\frac{\lambda c_{2}}{2(4 \alpha-1)}+\frac{\lambda^{2} c_{1}^{2}}{(1-3 \alpha)^{2}}(1-\mu)
$$

So, for $x=\left|c_{1}\right| \leq 1$,

$$
\left|a_{3}-\mu a_{2}^{2}\right| \leq A x^{2}+\frac{\lambda}{2|1-4 \alpha|} \equiv H(x)
$$

where $A=\lambda^{2}|1-\mu| /(1-3 \alpha)^{2}-\lambda / 2|1-4 \alpha|$ and

$$
\left|a_{3}-\mu a_{2}^{2}\right| \leq \begin{cases}H(1)=\frac{\lambda^{2}|1-\mu|}{(1-3 \alpha)^{2}}, & A \geq 0, \\ H(0)=\frac{\lambda}{2|1-4 \alpha|}, & A<0 .\end{cases}
$$

The upper bound is sharp due to the functions $f_{1}(z)=z(1-3 \alpha) /(1-3 \alpha+\lambda z)$ and $f_{2}(z)=z \cdot \sqrt{(1-4 \alpha) /\left(1-4 \alpha+\lambda z^{2}\right)}$.

Remark 3.4. By putting $\alpha=1 /(2-\gamma), 0 \leq \gamma<1$, we get the result from [10, Theorem 3].

\section{Acknowledgments}

The work on this paper was done under the Joint Research Project financed by The Ministry of Education and Science of the Republic of Macedonia (MESRM) (Project no. 17/1383/1) and The Scientific and Technical Research Council of Turkey (TUBITAK) (Project no. TBGA-U-105T056).

\section{References}

[1] T. Bulboacă and N. Tuneski, New criteria for starlikeness and strongly starlikeness, Mathematica (Cluj) 43(66) (2001), no. 1, 11-22 (2003).

[2] P. L. Duren, Univalent Functions, Fundamental Principles of Mathematical Sciences, vol. 259, Springer, New York, 1983.

[3] S. S. Miller and P. T. Mocanu, Differential subordinations and univalent functions, The Michigan Mathematical Journal 28 (1981), no. 2, 157-172.

[4] _ On some classes of first-order differential subordinations, The Michigan Mathematical Journal 32 (1985), no. 2, 185-195.

[5] _ Differential Subordinations. Theory and Applications, Monographs and Textbooks in Pure and Applied Mathematics, vol. 225, Marcel Dekker, New York, 2000.

[6] M. Obradowič and N. Tuneski, On the starlike criteria defined by Silverman, Zeszyty Naukowe Politechniki Rzeszowskiej. Matematyka 181 (2000), no. 24, 59-64 (2001).

[7] V. Ravichandran, M. Darus, and N. Seenivasagan, On a criteria for strong starlikeness, The Australian Journal of Mathematical Analysis and Applications 2 (2005), no. 1, article 6, 12.

[8] H. Silverman, Convex and starlike criteria, International Journal of Mathematics and Mathematical Sciences 22 (1999), no. 1, 75-79.

[9] V. Singh, On some criteria for univalence and starlikeness, Indian Journal of Pure and Applied Mathematics 34 (2003), no. 4, 569-577.

[10] V. Singh and N. Tuneski, On criteria for starlikeness and convexity of analytic functions, Acta Mathematica Scientia. Series B 24 (2004), no. 4, 597-602. 
8 Starlikeness and convexity of analytic functions

[11] N. Tuneski, On certain sufficient conditions for starlikeness, International Journal of Mathematics and Mathematical Sciences 23 (2000), no. 8, 521-527.

[12] On a criteria for starlikeness of analytic functions, Integral Transforms and Special Functions 14 (2003), no. 3, 263-270.

[13] - On the quotient of the representations of convexity and starlikeness, Mathematische Nachrichten 248/249 (2003), no. 1, 200-203.

Nikola Tuneski: Faculty of Mechanical Engineering, Ss. Cyril and Methodius University,

Karpoš II b.b., 1000 Skopje, Macedonia

E-mail address: nikolat@mf.edu.mk

Hüseyın Irmak: Department of Mathematics Education, Faculty of Education, Başkent University, Bağlica Campus, Bağlica, Etimesgut, 06530 Ankara, Turkey

E-mail address: hisimya@baskent.edu.tr 


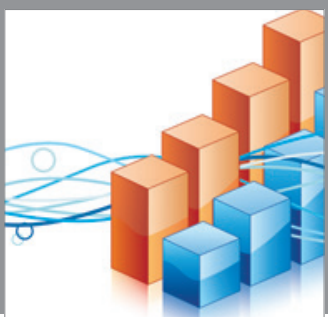

Advances in

Operations Research

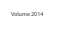

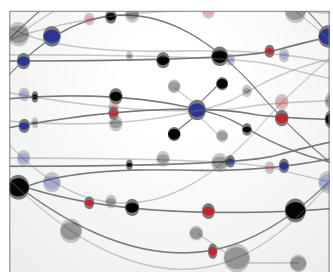

\section{The Scientific} World Journal
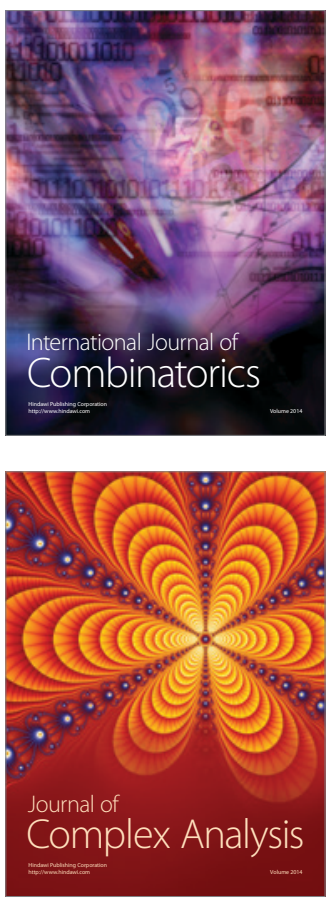

International Journal of

Mathematics and

Mathematical

Sciences
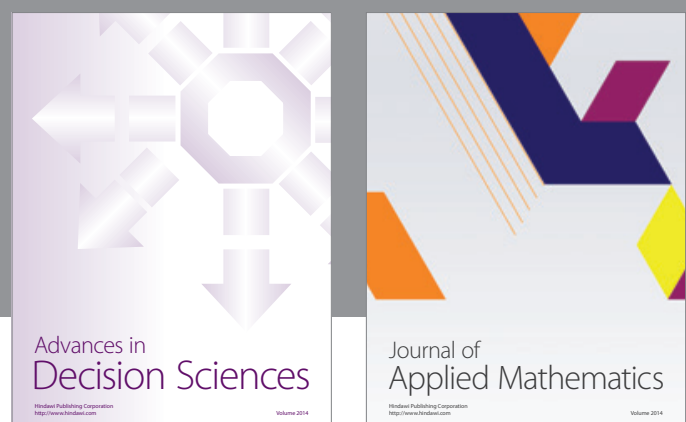

Journal of

Applied Mathematics
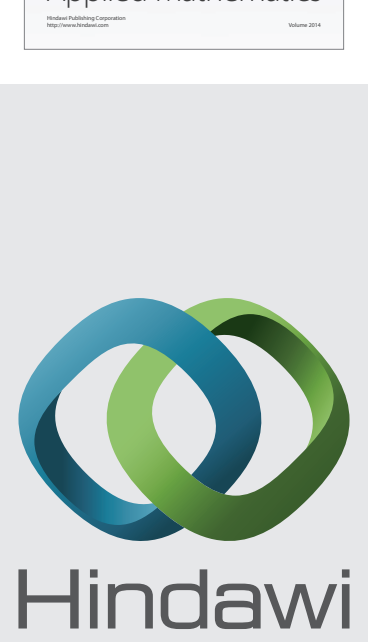

Submit your manuscripts at http://www.hindawi.com
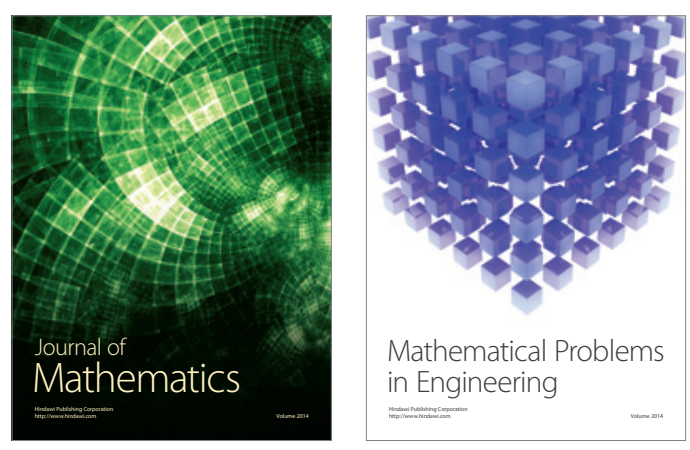

Mathematical Problems in Engineering
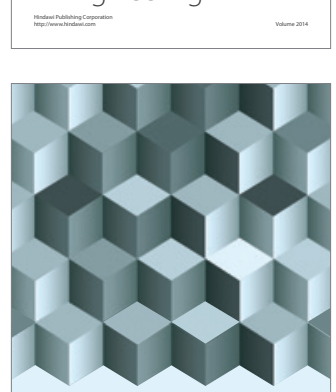

Journal of

Function Spaces
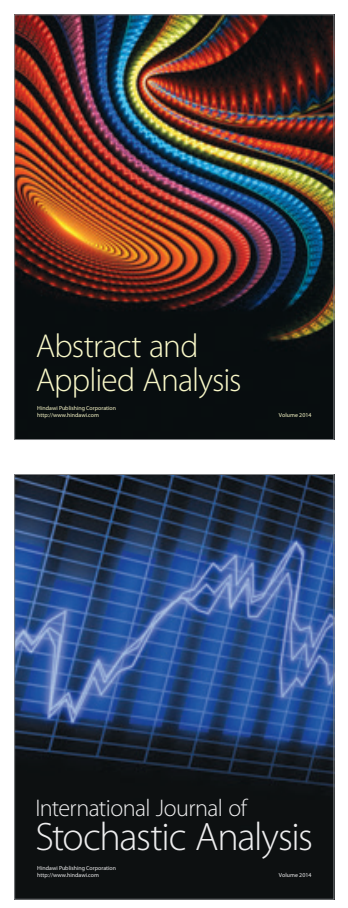

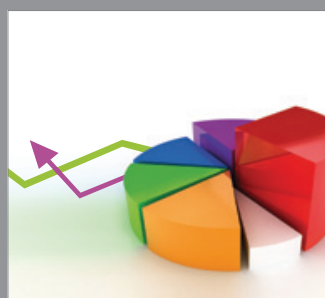

ournal of

Probability and Statistics

Promensencen
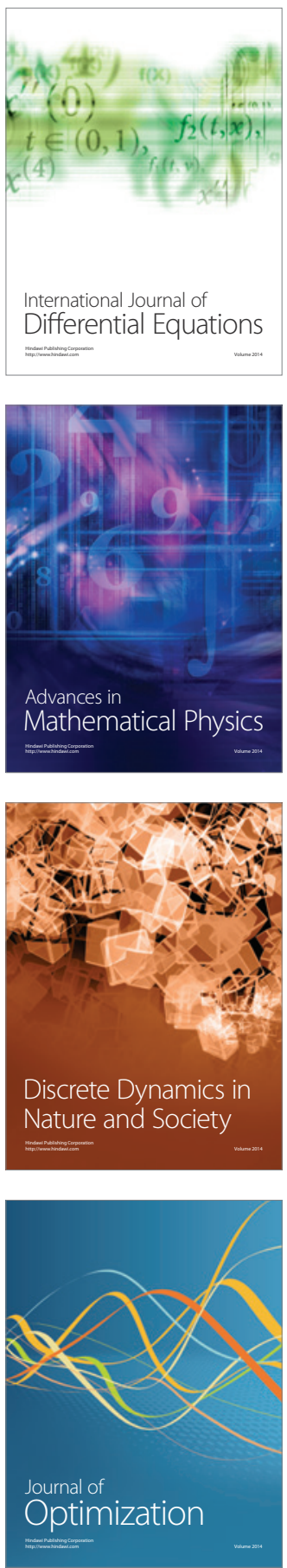